\title{
The association of antihypertensive use and depressive symptoms in a large older population with hypertension living in Australia and the United States: a cross-sectional study
}

\author{
Bruno Agustini ${ }^{1}$, Mohammadreza Mohebbi ${ }^{1,2}$, Robyn L. Woods ${ }^{3}$, John J. McNeil ${ }^{3}$, Mark R. \\ Nelson $^{4}$, Raj C. Shah ${ }^{5}$, Anne M. Murray ${ }^{6}$, Michael E. Ernst ${ }^{7,8}$, Christopher M. Reid ${ }^{3,9}$, \\ Andrew Tonkin ${ }^{3}$, Jessica E. Lockery ${ }^{3}$, Michael Berk ${ }^{1,10}$ ASPREE Investigator Group \\ ${ }^{1}$ School of Medicine, IMPACT Strategic Research Centre, Barwon Health, Deakin University, \\ Geelong, VIC, Australia \\ 2Biostatistics Unit, Deakin University, Geelong, VIC, Australia \\ ${ }^{3}$ School of Public Health and Preventive Medicine, Monash University, Melbourne, VIC, Australia \\ ${ }^{4}$ Menzies Institute for Medical Research, University of Tasmania, Hobart, TAS, Australia \\ ${ }^{5}$ Department of Family Medicine and Rush Alzheimer's Disease Center, Rush University Medical \\ Center, Chicago, IL, USA \\ ${ }^{6}$ Berman Center for Outcomes and Clinical Research, Hennepin Healthcare Research Institute, \\ Hennepin Healthcare, Minneapolis, MN, USA \\ ${ }^{7}$ Department of Pharmacy Practice and Science, College of Pharmacy, The University of lowa, \\ lowa, IA, USA \\ ${ }^{8}$ Department of Family Medicine, Carver College of Medicine, The University of lowa, lowa, IA, \\ USA \\ ${ }^{9}$ School of Public Health, Curtin University, Perth, WA, Australia \\ ${ }^{10}$ Orygen, The National Centre of Excellence in Youth Health, The Department of Psychiatry and \\ The Florey Institute for Neuroscience and Mental Health, University of Melbourne, Melbourne, \\ VIC, Australia
}

\section{Abstract \\ Cardiovascular drugs impact many pathways involved in depression pathophysiology and treatment. However, their distinct impact on mood is underrecognized and the literature is conflicting. Therefore, using a very large and well-characterised sample of older adults with hypertension, we aimed to investigate the prevalence of depressive symptoms in users of different}

Bruno Agustini, bagustini@deakin.edu.au.

Conflict of interest

The authors declare that they have no conflict of interest.

Supplementary information

The online version of this article (https://doi.org/10.1038/s41371-020-0303-y) contains supplementary material, which is available to authorized users.

Publisher's note Springer Nature remains neutral with regard to jurisdictional claims in published maps and institutional affiliations. 
antihypertensive classes. We analysed baseline data from 14,195 older individuals with hypertension enroled in a large clinical trial. Median age was 75 years. The association of antihypertensive use by class and depression prevalence, as measured by a validated depression scale, was determined using logistic regression models. Multivariable logistic models were implemented to account for important confounding factors. Our analyses showed a positive association between depressive symptoms and the use of beta blockers (BB) (OR: 1.37; 95\% CI: $1.17-1.60, p<0.01$ ), compared with users of other antihypertensive classes. All other classes of antihypertensives (including angiotensin-converting enzyme inhibitors, angiotensin-receptor blockers, and calcium channel blockers) were not significantly associated with depressive symptoms. In secondary analysis, this relationship was stronger for lipophilic (39\%) and nonselective BB (52\%) compared with hydrophilic (26\%) and selective medications (31\%), respectively. This study adds further evidence for a probable association between BB and depression in a large sample of older adults with hypertension and no history of cardiovascular disease or heart failure. These findings should regenerate interest and increase awareness of clinicians about the possible adverse effects of these medications in an otherwise healthy older population.

\section{Introduction}

Depression is highly prevalent among older adults. Following the rapid global increase in the population aged 60 years and older, the burden of late-life depression is expected to keep rising [1]. The prevalence and treatment rates of hypertension also tend to increase with age and hypertension is the most prevalent modifiable risk factor for cardiovascular diseases (CVD) in this population [2]. While the direct association of hypertension and depression is unclear [3], aged individuals tend to be more sensitive to medication adverse events and chronic conditions frequently cluster in this group, leading to multimorbidity and polypharmacy, with several medications potentially associated with depression as an adverse event [4]. Therefore, finding the best therapeutic strategies for treating hypertension in old age without increasing neuropsychiatric adverse events can improve clinical outcomes and significantly increase well being and the quality of life in this vulnerable population.

Depression is bidirectionally associated with cardiometabolic conditions and cardiovascular drugs impact many pathways related to depression pathophysiology [5]. Therefore, an important, yet underrecognized, role may exist for antihypertensive drugs in the development, treatment and/or prevention of mood disorders. However, studies of neuropsychiatric effects of these drugs have shown conflicting findings. While the some classes of antihypertensives, like angiotensin-converting enzyme inhibitors (ACEi) or angiotensin-receptor blockers (ARB), have been proposed as possible preventive or therapeutic strategies for depression [6,7], others like calcium channel blockers (CCB) and beta blockers $(\mathrm{BB})$ have been associated with an increase in depression incidence and prevalence $[4,8]$.

Hence, in this study we aim to investigate the association of different classes of antihypertensive medication with the presence of depressive symptoms in a very well- 
defined and large population of hypertensive older adults without a history of CVD or heart failure.

\section{Methods}

\section{Study population}

This cross-sectional study is part of a large population-based randomised controlled trial investigating the effects of aspirin on several endpoints in a healthy older population, the ASPirin in Reducing Events in the Elderly (ASPREE) [9]. ASPREE recruited a total of 19,114 participants from Australia and the United States. Inclusion criteria were communitydwelling men and women 70 years of age and older (US minority 65 years of age and older) who were willing and able to provide informed consent. Exclusion criteria included a history of cardiovascular event or established CVD or atrial fibrillation; dementia or a score of $<78$ on the Modified Mini-Mental State examination; the presence of significant disability; a condition with a high current or recurrent risk of bleeding, anaemia, a condition likely to cause death within 5 years; the current use of other antiplatelet or antithrombotic medication; the current use of aspirin for secondary prevention; and severe uncontrolled hypertension (i.e. systolic blood pressure (SBP) of $\geq 180 \mathrm{~mm} \mathrm{Hg}$ or a diastolic blood pressure (DBP) of $\geq 105 \mathrm{~mm} \mathrm{Hg}$ ). To address confounding by indication, in this study we excluded non-hypertensive participants $(n=4919)$, leaving a final sample of $14,195(74.2 \%)$ hypertensive individuals. Participants were recruited mainly from primary care services, with general practitioners as ASPREE co-investigators. Recruitment period went from 2010 to 2014. ASPREE recruitment strategies are described in detail elsewhere [9].

\section{Instruments and measures}

Sociodemographic questionnaires were administered at baseline, with information including age, education, gender, smoking status, alcohol use, living status, and self-reported presence and/or history of medical conditions. Participants had their height, weight (used to calculate body mass index-BMI), abdominal circumference, blood pressure, and heart rate measured. The presence of hypertension was defined as the mean of three blood pressure measurements performed by trained study staff using an automated blood pressure device with an appropriate sized cuff. A mean SBP $\geq 140 \mathrm{~mm} \mathrm{Hg}$ or DBP $\geq 90 \mathrm{~mm} \mathrm{Hg}$ or being on an antihypertensive medication defined participants as hypertensive. Blood samples were also collected, and the presence of diabetes was defined as an elevated fasting blood glucose ( $\geq 7 \mathrm{mmol} / \mathrm{L}$ ) or treatment for diabetes or self-report.

\section{Medication use}

Number and type of self-reported medication use were part of the baseline questionnaire and participants were asked to bring in their prescribed medications or a list of these. This information was utilised to indicate prevalent antihypertensive use, class and number of concomitant medications. Control groups were users of other classes of medication (primary hypothesis) and non-medicated hypertensive participants. Diuretic users (no previous report or plausible biological mechanism for an association with depression) were included in the second control group alongside non-medicated hypertensive individuals $(n=4747)$. 


\section{Depression measurement}

The Centre for Epidemiological Studies Depression (CES-D 10) scale was used to quantify the presence of depressive symptoms [10]. The CES-D 10 is a self-completed questionnaire that scores the severity of depressive symptoms "during the past week" on a four-point (0-3) scale, providing a score that ranges from 0 to 30 . This instrument has shown comparable accuracy to the full version of the CES-D $(\kappa=0.97)$ in classifying participants with depressive symptoms [10]. Construct validity of the CES-D 10 showed that a single score was a reliable and the valid measure of depression in an older population [11]. When compared with a formal psychiatric diagnosis of late-life depression, the scale was shown to have a sensitivity of $97 \%$ and a specificity of $84 \%$ [12]. Although not a formal psychiatric diagnosis, the presence of minor and/or subthreshold depressive symptoms has been shown to significantly impact the quality of life and the prognosis in older adults [13, 14]. Following the previous research, a cutoff of $\geq 8$ was defined as positive screening for the presence of important depressive symptoms [15]. Based on the literature, we use the term "clinically relevant depressive symptoms" (CRD) as synonymous of positive screening according to our threshold [16].

\section{Statistical analysis}

Baseline characteristics of participants according to antihypertensive use were compared using independent sample $t$-tests for continuous measures or chi-square tests for categorical measures. The association of antihypertensive use with dichotomised depressive symptoms was determined using logistic regression models and odds ratios (ORs) and 95\% confidence intervals (CIs) are reported. Based on the prevalence of CRD in this whole population being $<10 \%$, we assumed that the estimated OR's are a close approximate of the prevalence ratios [17].

The following analyses were specified prior to the examination of data: (1) the association of CRD with each drug class compared with users of other classes of medication; (2) the association of CRD with each class of antihypertensive medication with non-medicated hypertensives (and diuretic users) as controls; (3) the association of CRD with combination treatments and number of medications. In exploratory analyses, we investigated the association of CRD and BB individually and divided according to their $\beta$-receptor selectivity and relative lipophilicity, thought to modulate the effects of these drugs in mood symptoms [18].

Multivariable logistic models were implemented to account for sociodemographic factors associated with depression, namely gender, education, smoking, and living status. To avoid multicollinearity between metabolic factors, stratified analyses were performed for gender, age, BMI, diabetes, uncontrolled hypertension, and abdominal circumference. Subgroup analysis according to BP levels and antidepressant use were also included. All statistical tests were two-tailed, and $p$ value of $<0.05$ was considered to indicate statistical significance. All analyses were performed in the ASPREE baseline dataset, using STATA software, version 15.0. 


\section{Results}

Table 1 shows characteristics of participants according to the class of antihypertensive drug use. The mean age was 75 years (SD: 4.5 years). A total number of 14,195 (74.2\% of original sample) participants were classified with hypertension and included in the analyses. Of those, 7922 (55.8\%) were female. The prevalence of hypertension tended to increase with age (72\% in 65-74 years old to $83 \%$ in individuals with 85 years or more) and 1802 individuals had comorbid diabetes mellitus (12.69\%) (Table 1).

Overall 1414 participants (9.9\%) had a CES-D 10 score of $\geq 8$ and were classified as having CRD. Depressive symptoms were more prevalent in females $(n=931 ; 11.7 \%)$ when compared with males $(n=483 ; 7.7 \%)$; individuals educated for 12 years or less, living alone or in a residential care facility, and in ethnic minorities and current smokers. Current alcohol use was not statistically significantly associated with depressive symptoms. Antidepressants were used by 1624 individuals (11.4\%).

Of all individuals classified as hypertensive, $66.5 \%$ were on at least one medication for hypertension (excluding diuretics) (Table 1). Medicated groups did not differ significantly regarding mean SBP and DBP or the prevalence of uncontrolled hypertension. However, the non-medicated group tended to have higher mean blood pressure levels, higher percentage of males, more educated, younger and fitter individuals (Table 1). Conversely, BB users had a lower heart rate, included the largest percentage of females, living alone, severely obese and tended to use more concomitant medications (Table 1).

Logistic regression models (Table 2) showed a statistically significant association of BB use and a $46 \%$ increased prevalence of CRD when compared with other classes of medication in the unadjusted model. The association remained significant after adjustment for multiple confounders (OR: 1.37; 95\% CI: 1.17-1.60, $p<0.01$ ). In separate analysis, BB use was associated with increased CRD when compared with non-medicated controls, but there was only a trend (OR: 1.28, $p=0.08$ ) in the adjusted models (Supplement 1 ).

All other classes of antihypertensive medications (i.e. ACEi, ARB, and CCB) were not significantly associated with the prevalence of depressive symptoms in the unadjusted and adjusted models (Table 2), as well as in individual drug analyses.

Combination treatment was present in $27.9 \%$ of the hypertensive population $(n=3968)$. Of all possible combinations, only the combination of $\mathrm{ARB}$ and $\mathrm{BB}$ was significantly associated with depressive symptoms (ARB + BB: OR 1.62, 95\% CI 1.18-2.22, $p<0.01$ ). All possible combined treatments were analysed. A number of participants and their associations with depressive symptoms in multivariate models are presented in Supplement 2.

Association between antihypertensive use and depressive symptoms by subgroup analyses were performed for all medication classes, but only the ones involving BB were shown to be significant. BB use was associated with increased depressive symptoms in all subgroups, except for males, individuals with diabetes and $>85$ years of age, possibly because of power issue in those groups (Table 3). 
Exploratory analyses of BB according to lipophilicity and $\beta$-receptor selectivity were also performed. When grouped together, lipophilic BB were associated with a 39\% increase in the prevalence of CRD (95\% CI: $1.10-1.75 ; p<0.01$ ) versus a $26 \%$ (95\% CI 1.03-1.56; $p=$ $0.02)$ increase in the hydrophilic group. When divided according to $\beta$-selectivity, selective BB were associated with 31\% (95\% CI: 1.10-1.56; $p<0.01$ ) increased odds of CRD against a 52\% (95\% CI: $1.08-2.13 ; p=0.01)$ increase in the non-selective group. In individual medication analysis, metoprolol was significantly associated with higher prevalence of CRD and there was a trend for this association for propranolol and atenolol (Table 4). Other BB were not analysed individually due to the small sample size (Supplement 3).

\section{Discussion}

In this cross-sectional study of a well-characterised sample of over 14,000 hypertensive older adults without a history of CVD or heart failure, the use of BB was significantly associated with an increased prevalence of depressive symptoms. The association persisted after controlling for important confounding factors. On secondary analyses, lipophilic and non-selective BB appear to be more strongly associated with increased CRD prevalence in comparison with hydrophilic and $\beta 1$-selective medications, respectively. Other classes of antihypertensive treatment had no association with depressive symptoms (except when ARB was used in combination with a BB). These results add further evidence to the controversial topic of BB use and depressive symptoms in a sample of otherwise healthy older individuals with hypertension.

Much of the controversy regarding BB use and depression might be explained by heterogeneity in studies' populations. Studies, including reviews and meta-analyses, that found a protective effect of BB or sno association with depressive symptoms have often included participants with a history of CVD, myocardial infarction or heart failure and distinct age groups [19-25]. In these populations, the impact of BB on improved health may outweigh or confound its effect on mood.

Our results are consistent with the findings of the only study conducted in a primary care setting of healthy (no CVD or heart failure) hypertensive older adults from the Netherlands $(n=573$; mean age $=70)$ [26]. In this cross-sectional study, the use of lipophilic BB was significantly associated with increased prevalence of depressive symptoms after adjustment for multiple confounders (OR: 1.60; 95\% CI: 1.08-2.36). The study had a very similar design, but it was underpowered to test the effects of hydrophilic $\mathrm{BB}(n=13)$, included in the control group. This result also agrees with a prospective study of 5104 older adults from the Rotterdam Study, in which the use of lipophilic BB was associated with an increased risk of incident depression (especially in the first 3 months of use) [27]. However, this study included participants with established CVD and therefore is not representative of our population.

Several possible mechanisms have been reported to account for the possible association of BB and depression, including an impact of these agents on sleep [28], circadian regulation [29], and sympathetic-mediated feedback loops essential for energy regulation and emotional arousal [30, 31]. In addition, Table 1 shows that BB users tend to include more 
females and individuals who reported living alone, factors previously associated with depression in this population [15]. However, the association persisted after we controlled for these factors in the multivariate models.

No other classes of antihypertensive drugs were associated with the reduced or increased prevalence of CRD. Drugs that act on the renin-agiotensin system have been associated with the decreased risk of hospitalisations for mood disorders [32], lower frequency of antidepressant consumption [33], and increased quality of life [7]. Again, heterogeneity in studies, especially related to age differences, might explain some of the discrepancies. Plasma renin activity is significantly reduced after 60 years of age, as is aldosterone, kidney and adrenal gland function $[34,35]$, probably diminishing the effects of these drugs in this system.

Regarding mood disorders, CCB have been mainly explored for bipolar disorder and a cohort study found benefits for their use as an adjunctive therapy in the treatment of depression and associated cognitive dysfunction [36]. On the other hand, their use as monotherapy for hypertension was associated with increased hospitalisation for mood disorders [32]. An ongoing trial of CCB focusing on mood symptoms and cognition might provide more evidence for this in the near future [37].

The strengths of this study include a much larger and well-characterised sample of community-dwelling older adults compared with previous studies, a comprehensive assessment of individuals, well-validated instruments for depression assessment, and a strict protocol of three BP measures taken by trained professionals. The exclusion of nonhypertensive participants significantly contributes to reduce confounding by indication effects, although these cannot be excluded with this study design [38]. Accounting for a variety of confounders, including metabolic and sociodemographic factors previously associated with depression, in well-powered multivariable models was another advantage.

There are also some limitations. First, due to its cross-sectional design, only association, and not causation, can be inferred. Also, notwithstanding the fact that the CES-D 10 is a valuable tool for depression screening, it is not a formal diagnostic tool for depression, hence why we used "CRD" instead of "depression" while citing results. Nevertheless, the presence of subthreshold depressive symptoms significantly impacts the quality of life in old age, making this a reliable instrument for screening $[11,13,14]$. The duration of therapy, primary indication, doses and adherence to antihypertensive drugs might also interfere in their relationship with mood. Unfortunately, we do not have data on those. BP levels are highly influenced by stress and even with three measures, this is not the gold-standard, especially in stress-prone individuals. Another limitation comes from exclusion criteria. Since the ASPREE study excluded subjects with severe diseases, dementia, uncontrolled hypertension, and especially, individuals with a history of CVD or heart failure, these subgroups were not addressed in this study. BB have an important role in reducing mortality in CVD and heart failure [39] and do not appear to be associated with increased prevalence of depression in this population $[19,20]$. Because our sample is composed exclusively by older adults without a history of CVD, extrapolation of our results to the general population must take that into consideration. 


\section{Conclusion}

This study provides further evidence for a possible association between BB use and increased prevalence of depressive symptoms in a large population of community-dwelling hypertensive older adults. The association persisted after controlling for important confounding factors. There was no association of other antihypertensive drug classes and the prevalence of depressive symptoms. Guidelines on hypertension screening and treatment, progressively set more sensitive for treatment and targets, should consider the possible neuropsychiatric adverse events of these drugs. Despite no longer being recommended as first-line treatment for hypertension in older individuals [2, 40, 41] these drugs remain highly prescribed for this indication. Medication reviews are an important part of older adults' care and deprescribing efforts should be taken if medication adverse effects in mood are suspected. Depression is an important factor to consider while prescribing for older populations, requiring clinicians to balance between mental health and quality of life and possible gains in morbidity and mortality. Therefore, depression merits being in the mind of attending physicians, guideline writers and policy makers.

\section{Supplementary Material}

Refer to Web version on PubMed Central for supplementary material.

\section{Acknowledgements}

The authors acknowledge the efforts of research personnel and long-term involvement of participants of the ASPREE Study.

Funding

The study is supported by the National Institute on Aging and the National Cancer Institute at the National Institutes of Health (grant number U01AG029824); the National Health and Medical Research Council of Australia (grant numbers 334047 and 1127060); Monash University (Australia); the Victorian Cancer Agency (Australia). MB is supported by a NHMRC Senior Principal Research Fellowship (APP1059660 and APP1156072) and CMR is supported by a NHMRC Senior Research Fellowship (APP1045862).

\section{References}

1. World Health Organization. Depression and other common mental disorders: global health estimates. Geneva World Heal Organ. 2017;1-24. http://apps.who.int/iris/bitstream/ 10665/254610/1/WHOMSD-MER-2017.2-eng.pdf.

2. Pimenta E, Oparil S. Management of hypertension in the elderly. Nat Rev Cardiol. 2012;9:286-96. 10.1038/nrcardio.2012.27. [PubMed: 22411292]

3. Chen L, Li Y, Chen P, Hu Y, Li Z. Prevalence of depression in patients with hypertension. Medicine. 2015;94:e1317. [PubMed: 26252317]

4. Qato DM, Ozenberger K, Olfson M. Prevalence of prescription medications with depression as a potential adverse effect among adults in the United States. JAMA. 2018;319:2289 10.1001/ jama.2018.6741. [PubMed: 29896627]

5. Amare AT, Schubert KO, Klingler-Hoffmann M, Cohen-Woods S, Baune BT. The genetic overlap between mood disorders and cardiometabolic diseases: a systematic review of genome wide and candidate gene studies. Transl Psychiatry. 2017;7:e1007-12. 10.1038/tp.2016.261. [PubMed: 28117839]

6. Vian J, Pereira C, Chavarria V, Köhler C, Stubbs B, Quevedo J, et al. The renin-angiotensin system: a possible new target for depression. BMC Med. 2017;15:1-13. [PubMed: 28049467] 
7. Brownstein DJ, Salagre E, Köhler C, Stubbs B, Vian J, Pereira C, et al. Blockade of the angiotensin system improves mental health domain of quality of life: A meta-analysis of randomized clinical trials. Aust NZ J Psychiatry. 2018;52:24-38.

8. Wilson DL, Ried LD. Identifying iatrogenic depression using confirmatory factor analysis of the Center for Epidemiologic Studies Depression Scale in patients prescribed a verapamil-sustainedrelease-led or atenolol-led hypertension treatment strategy. Res Soc Adm Pharm. 2012;8:309-20. 10.1016/j.sapharm.2011.08.002.

9. Grimm R, McNeil JJ, Applegate W, Beilin L, Espinoza S, Johnston CI, et al. Study design of ASPirin in Reducing Events in the Elderly (ASPREE): a randomized, controlled trial. Contemp Clin Trials. 2013;36:555-64. 10.1016/j.cct.2013.09.014. [PubMed: 24113028]

10. Andresen EM, Malmgren JA, Carter WB, Patrick DL. Screening for depression in well older adults: evaluation of a short form of the CES-D (Center for Epidemiologic Studies Depression Scale). Am J Prev Med. 1993;10:77-84. http://www.ncbi.nlm.nih.gov/pubmed/8037935.

11. Mohebbi M, Nguyen V, McNeil JJ, Woods RL, Nelson MR, Shah RC, et al. Psychometric properties of a short form of the Center for Epidemiologic Studies Depression (CES-D-10) scale for screening depressive symptoms in healthy community dwelling older adults. Gen Hosp Psychiatry. 2017;1-9. 10.1016/j.genhosppsych.2017.08.002.

12. Irwin M, Artin KH, Oxman M. Screening for depression in the older adult. Arch Intern Med. 1999;159:1701-4. [PubMed: 10448771]

13. Da Silva SA, Scazufca M, Menezes PR. Population impact of depression on functional disability in elderly: results from "são Paulo Ageing \& Health Study" (SPAH). Eur Arch Psychiatry Clin Neurosci. 2013;263:153-8. [PubMed: 22872105]

14. Cuijpers P, Vogelzangs N, Twisk J, Kleiboer A, Li J, Penninx BW. Differential mortality rates in major and subthreshold depression: meta-analysis of studies that measured both. Br J Psychiatry. 2013;202:22-7. [PubMed: 23284149]

15. Mohebbi M, Agustini B, Woods RL, McNeil JJ, Nelson MR, Shah RC, et al. Prevalence of depressive symptoms and its associated factors among healthy community-dwelling older adults living in Australia and the United States. International journal of geriatric psychiatry, 2019;34:1208-16. [PubMed: 30989707]

16. Miszkurka M, Zunzunegui MV, Ylli A, Deshpande N, Guralnik J, Phillips SP. Clinically relevant depression in old age: an international study with populations from Canada, Latin America and Eastern Europe. Psychiatry Res. 2016;241:236-41. 10.1016/j.psychres.2016.04.096. [PubMed: 27183110]

17. Alexander Persoskie, Ferrer RA. A most odd ratio: interpreting and describing odds ratios. Am J Prev Med. 2017;52:224-8. [PubMed: 27639787]

18. Poirier L, Tobe SW. Contemporary use of $\beta$-blockers: clinical relevance of subclassification. Can $\mathbf{J}$ Cardiol. 2014;30:9-15.

19. Battes LC, Regar E, Al Amri I, de Jaegere PPT, Pedersen SS, Oemrawsingh RM, et al. Beta blocker therapy is associated with reduced depressive symptoms 12 months post percutaneous coronary intervention. J Affect Disord. 2011;136:751-7. 10.1016/j.jad.2011.09.047. [PubMed: 22032873]

20. Hoogwegt MT, Kupper N, Theuns DAMJ, Jordaens L, Pedersen SS. Beta-blocker therapy is not associated with symptoms of depression and anxiety in patients receiving an implantable cardioverterdefibrillator. Europace. 2012;14:74-80. [PubMed: 21920910]

21. Gerstman BB, Heidi MJ, Mark B, Pyone C, James ML, Richard P. The incidence of depression in new users of beta-blockers and selected antihypertensives. J Clin Epidemiol. 1996;49:809-15. http://linkinghub.elsevier.com/retrieve/pii/0895435696000170?showall=true. [PubMed: 8691233]

22. Ko DT, Hebert PR, Coffey CS, Sedrakyan A, Curtis JP and Krumholz HM. $\beta$-blocker therapy and symptoms of depression, fatigue, and sexual dysfunction. Jama, 2002;288:351-7. [PubMed: 12117400]

23. Johansen A, Holmen J, Stewart R, Bjerkeset O. Anxiety and depression symptoms in arterial hypertension: the influence of antihypertensive treatment. The HUNT study, Norway. Eur J Epidemiol. 2012;27:63-72. [PubMed: 22183137] 
24. Ranchord AM, Spertus JA, Buchanan DM, Gosch KL, Chan PS. Initiation of $\beta$-blocker therapy and depression after acute myocardial infarction. Am Heart J. 2016;174:37-42. 10.1016/ j.ahj.2015.11.018. [PubMed: 26995368]

25. Verbeek DEP, Van Riezen J, De Boer RA, Van Melle JP, De Jonge P. A review on the putative association between beta-blockers and depression. Heart Fail Clin. 2011;7:89-99. 10.1016/ j.hfc.2010.08.006. [PubMed: 21109212]

26. Ringoir L, Pedersen SS, Widdershoven JW, Pouwer F, Keyzer JM, Romeijnders AC et al. Betablockers and depression in elderly hypertension patients in primary care. Family Medicine, 2014;46:447-53. [PubMed: 24911300]

27. Luijendijk HJ, Van Den Berg JF, Hofman A, Tiemeier H, Stricker BHC. $\beta$-Blockers and the risk of incident depression in the elderly. J Clin Psychopharmacol. 2011;31:45-50. [PubMed: 21192142]

28. Stoschitzky K, Sakotnik A, Lercher P, Zweiker R, Maier R, Liebmann P, et al. Influence of betablockers on melatonin release. Eur J Clin Pharmacol. 1999;55:111-5. [PubMed: 10335905]

29. Tahara Y, Takatsu Y, Shiraishi T, Kikuchi Y, Yamazaki M, Motohashi H, et al. Age-related circadian disorganization caused by sympathetic dysfunction in peripheral clock regulation. npj Aging and Mechanisms of Disease, 3;2017:1-11. [PubMed: 28649419]

30. Leentjens AFG. Neurobiological correlates of emotional processing in Parkinson's disease: a systematic review of experimental studies. J Psychosom Res. 2017;100:65-76. 10.1016/ j.jpsychores.2017.07.009. [PubMed: 28789795]

31. Harrell CS, Gillespie CF, Neigh GN. Energetic stress: the reciprocal relationship between energy availability and the stress response. Physiol Behav. 2016;166:43-55. 10.1016/ j.physbeh.2015.10.009. [PubMed: 26454211]

32. McCallum L, Boal AH, Muir S, Touyz RM, Smith DJ, Dominiczak AF, et al. Monotherapy with major antihypertensive drug classes and risk of hospital admissions for mood disorders. Hypertension.2016;68:1132-8. [PubMed: 27733585]

33. Nasr SJ, Crayton JW, Agarwal B, Wendt B, Kora R. Lower frequency of antidepressant use in patients on renin-angiotensin-aldosterone system modifying medications. Cell Mol Neurobiol. 2011;31:615-8. [PubMed: 21301954]

34. Lionakis N, Mendrinos D, Sanidas E, Favatas G, Georgopoulou M. Hypertension in the elderly. World J Cardiol. 2012;26:135-47.

35. Ntourakis D, Agouridis AP, Johnson EO, Tsioutis C, Yiallouris A, Agapidaki E, et al. Adrenal aging and its implications on stress responsiveness in humans. Front Endocrinol. 2019;10:1-12.

36. Tully PJ, Peters R, Pérès K, Anstey KJ and Tzourio C Effect of SSRI and calcium channel blockers on depression symptoms and cognitive function in elderly persons treated for hypertension: three city cohort study. International psychogeriatrics, 2018;30:1345-54. [PubMed: 29559030]

37. Atkinson LZ, Colbourne L, Smith A, Harmer CH, Nobre AC, Rendell J, et al. The Oxford study of Calcium channel Antagonism, Cognition, Mood instability and Sleep (OxCaMS): study protocol for a randomised controlled, experimental medicine study. Trials, 2019;20; p.120. [PubMed: 30755265]

38. Agustini B and Berk M Medications with depression as an adverse effect. Jama, 2018;320:1815.

39. Bangalore S, Sawhney S, Messerli FH. Relation of beta-blocker-induced heart rate lowering and cardioprotection in hypertension. J Am Coll Cardiol. 2008;52:1482-9. [PubMed: 19017516]

40. Parekh N, Page A, Ali K, Davies K, Rajkumar C. A practical approach to the pharmacological management of hypertension in older people. Ther Adv Drug Saf. 2017;8:117-32. [PubMed: 28439398]

41. Lindholm LH, Carlberg B, Samuelsson O. Should $\beta$ blockers remain first choice in the treatment of primary hypertension? A meta-analysis. Lancet. 2005;366:1545-53. [PubMed: 16257341] 


\section{Summary}

\section{What is known about the topic}

- Cardiovascular drugs impact many pathways involved in the pathophysiology of depression.

- Different classes of antihypertensive medication might have either beneficial or deleterious effects on mood.

- Mood effects of these drugs are not well described in an older population with no established cardiovascular disease.

\section{What this study adds}

- $\quad$ These findings add further evidence for an association between beta-blockers and depressive symptoms in older adults treated for uncomplicated hypertension.

- $\quad$ Other classes of antihypertensive medication had no association with depression, suggesting a safer neuropsychiatric profile for their use in the treatment of hypertension in otherwise healthy older adults. 


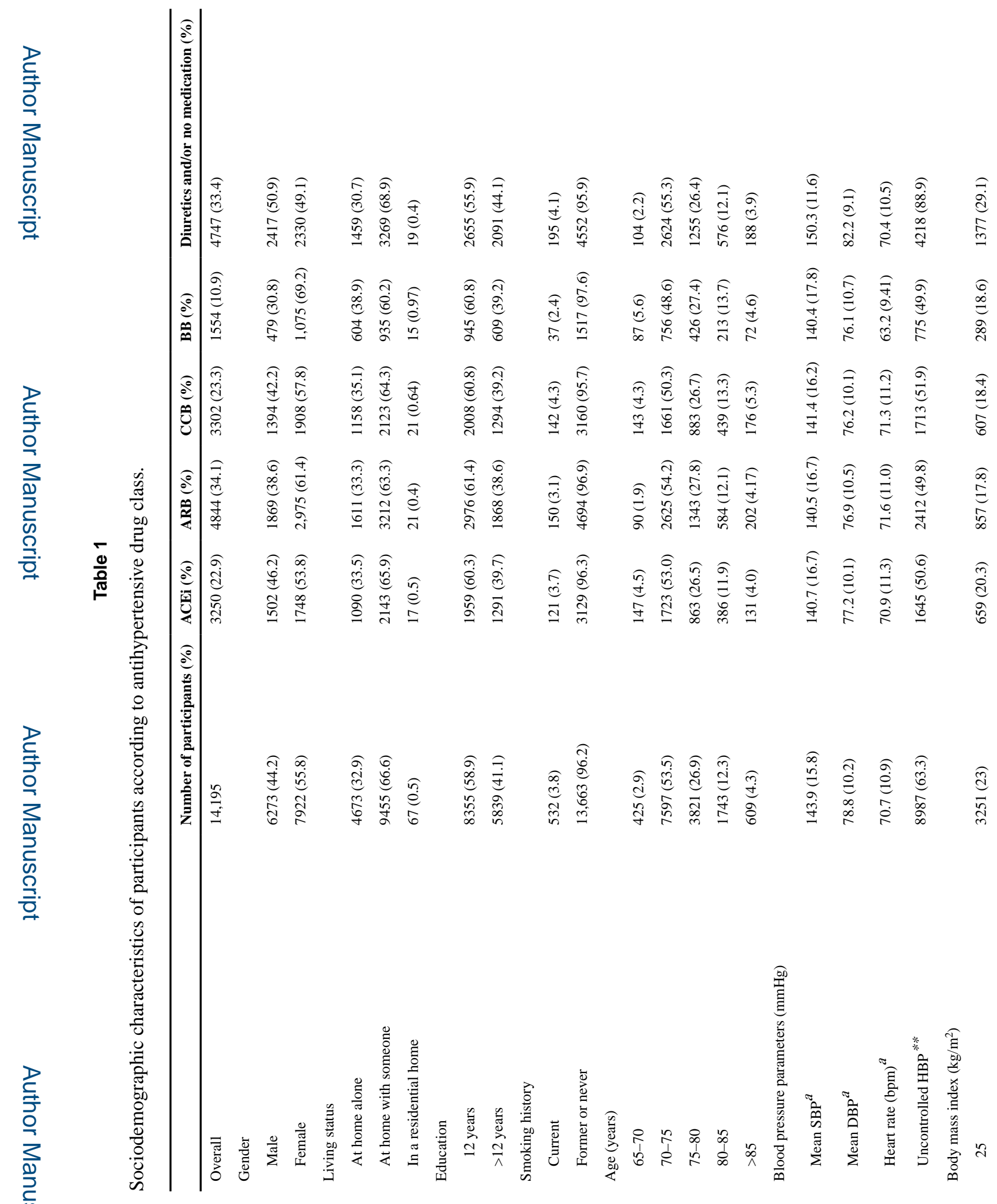

J Hum Hypertens. Author manuscript; available in PMC 2020 November 14. 
Agustini et al.

Page 13

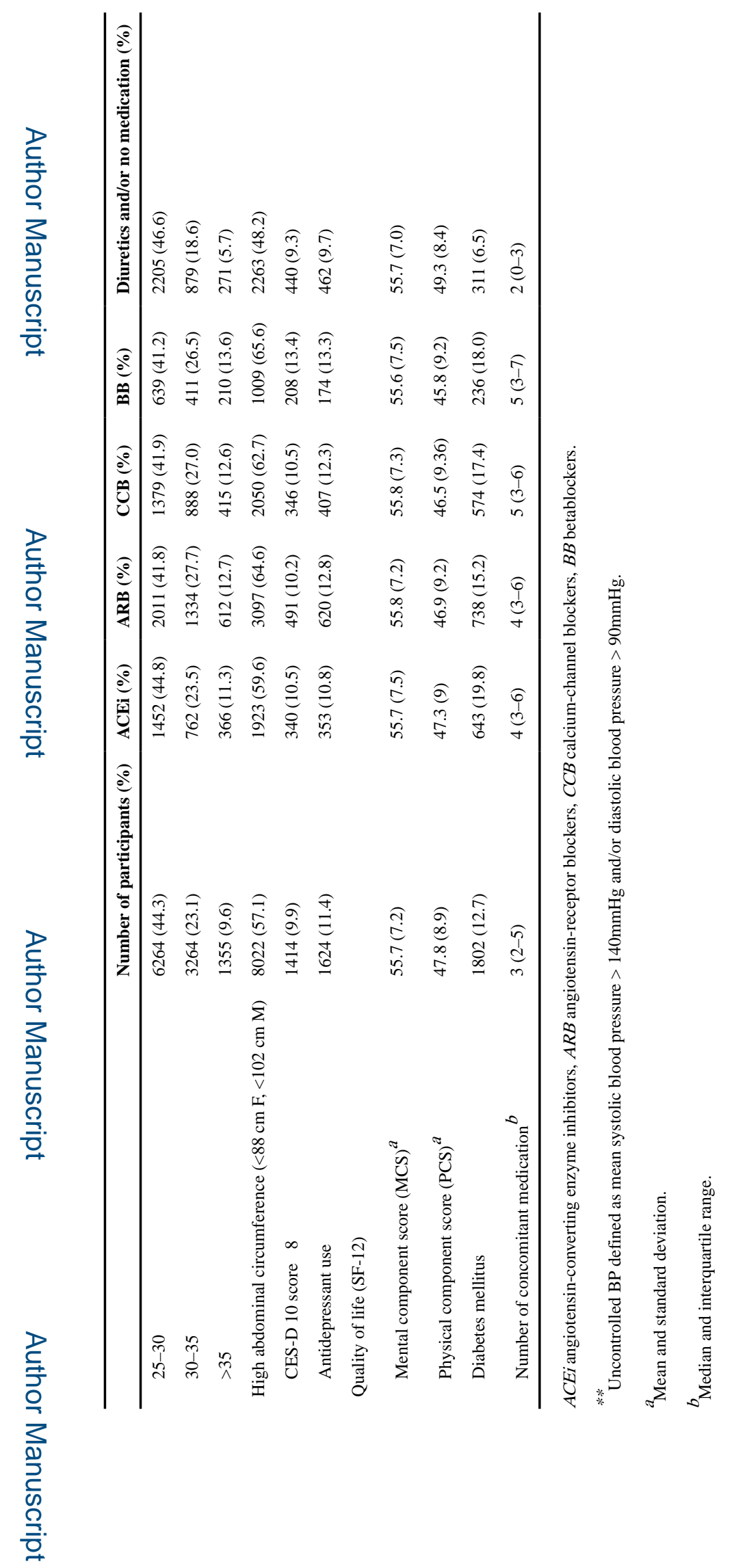

J Hum Hypertens. Author manuscript; available in PMC 2020 November 14. 


\section{Table 2}

Examining the association between the use of antihypertensive drug class and depressive symptoms (CES-D $\geq$ 8) with users of other classes and non-medicated as controls.

\begin{tabular}{|c|c|c|c|}
\hline & OR & $\mathbf{9 5 \%}$ confidence intervals & $p$ value \\
\hline \multicolumn{4}{|c|}{ Unadjusted } \\
\hline ACEi & 1.07 & $0.94-1.22$ & 0.27 \\
\hline ARB & 1.03 & $0.92-1.16$ & 0.61 \\
\hline $\mathrm{CCB}$ & 1.08 & $0.95-1.22$ & 0.26 \\
\hline BB & 1.46 & $1.25-1.71$ & $<0.01$ \\
\hline \multicolumn{4}{|c|}{ Gender adjusted } \\
\hline ACEi & 1.09 & $0.95-1.24$ & 0.20 \\
\hline ARB & 0.99 & $0.88-1.11$ & 0.88 \\
\hline $\mathrm{CCB}$ & 1.06 & $0.94-1.21$ & 0.34 \\
\hline BB & 1.37 & $1.17-1.61$ & $<0.001$ \\
\hline \multicolumn{4}{|c|}{ Age and gender adjusted } \\
\hline ACEi & 1.09 & $0.95-1.24$ & 0.20 \\
\hline ARB & 0.99 & $0.88-1.11$ & 0.88 \\
\hline $\mathrm{CCB}$ & 1.06 & $0.94-1.21$ & 0.34 \\
\hline BB & 1.37 & $1.17-1.61$ & $<0.001$ \\
\hline \multicolumn{4}{|c|}{ Multivariate analysis $^{a}$} \\
\hline ACEi & 1.08 & $0.95-1.23$ & 0.24 \\
\hline ARB & 0.99 & $0.89-1.12$ & 0.94 \\
\hline $\mathrm{CCB}$ & 1.05 & $0.92-1.19$ & 0.49 \\
\hline BB & 1.37 & $1.16-1.60$ & $<0.001$ \\
\hline
\end{tabular}

$A C E i$ angiotensin-converting enzyme inhibitors, $A R B$ angiotensin-receptor blockers, $C C B$ calcium channel blockers, $B B$ beta blockers.

${ }^{a}$ Accounting for gender, living status, education and smoking history (all significant at 0.05 level). Non-significant variables were excluded from multivariable models using a backward elimination method. 


\section{Table 3}

Subgroup analyses on the association between beta blocker use and depression accounting for age, metabolic conditions, uncontrolled hypertension, antidepressant use, blood pressure and heart rate levels, with users of other classes and non-medicated as controls.

\begin{tabular}{|c|c|}
\hline Subgroup analysis for BB users (n) & CES-D $\geq 8 \mathrm{MOR}^{a} ;(95 \%$ confidence intervals); $p$ value \\
\hline \multicolumn{2}{|l|}{ Age (years) } \\
\hline$\leq 74(8021)$ & $1.45(1.18-1.79) ;<0.01$ \\
\hline $75-84(5562)$ & $1.29(1.00-1.66) ; 0.05$ \\
\hline $85+(609)$ & $1.04(0.47-2.31) ; 0.92$ \\
\hline \multicolumn{2}{|l|}{ Gender } \\
\hline Male (6272) & $1.30(0.94-1.79) ; 0.10$ \\
\hline Female (7920) & $1.38(1.15-1.66) ;<0.01$ \\
\hline \multicolumn{2}{|l|}{ BMI $\left(\mathrm{kg} / \mathrm{m}^{2}\right)$} \\
\hline 소 (3251) & $1.73(1.22-2.46) ;<0.01$ \\
\hline $25-30$ (6226) & 1.27 (0.99-1.65); 0.06 \\
\hline $30-35$ (3263) & $1.54(1.14-2.09) ;<0.01$ \\
\hline $35+(1355)$ & $0.87(0.55-1.36) ; 0.53$ \\
\hline \multicolumn{2}{|l|}{ Diabetes } \\
\hline Yes (1790) & $1.30(0.90-1.89) ; 0.16$ \\
\hline No $(12,391)$ & $1.35(1.14-1.62) ;<0.01$ \\
\hline \multicolumn{2}{|l|}{ Antidepressant use } \\
\hline Yes (1624) & 1.44 (1.03-2.01); 0.03 \\
\hline No $(12,568)$ & $1.33(1.11-1.60) ;<0.01$ \\
\hline \multicolumn{2}{|l|}{ Uncontrolled hypertension $^{b}$} \\
\hline Yes (8986) & 1.34 (1.06-1.67); 0.01 \\
\hline No (5206) & $1.36(1.08-1.71) ;<0.01$ \\
\hline \multicolumn{2}{|l|}{ Abdominal circumference } \\
\hline Normal (6033) & $1.46(1.10-1.93) ;<0.01$ \\
\hline High (8021) & $1.31(1.08-1.59) ;<0.01$ \\
\hline \multicolumn{2}{|l|}{ SBP levels (mmHg) } \\
\hline$\leq 10(292)$ & $0.98(0.42-2.26) ; 0.96$ \\
\hline $111-140(5135)$ & $1.39(1.10-1.75) ;<0.01$ \\
\hline $141-160(6670)$ & $1.35(1.03-1.78) ; 0.03$ \\
\hline$>160(2098)$ & $1.29(0.85-1.97) ; 0.22$ \\
\hline \multicolumn{2}{|l|}{ DBP levels (mmHg) } \\
\hline$\$ 80(8015)$ & $1.47(1.20-1.79) ;<0.01$ \\
\hline $81-90(4323)$ & $1.12(0.80-1.57) ; 0.49$ \\
\hline $91-100(1645)$ & $1.46(0.87-2.44) ; 0.15$ \\
\hline$>100(212)$ & $1.43(0.42-4.86) ; 0.56$ \\
\hline \multicolumn{2}{|l|}{ Heart rate (beats/min) } \\
\hline$\$ 60(2424)$ & $1.38(1.02-1.86) ; 0.03$ \\
\hline $61-70(4972)$ & $1.26(0.96-1.65) ; 0.09$ \\
\hline
\end{tabular}

J Hum Hypertens. Author manuscript; available in PMC 2020 November 14. 


\begin{tabular}{ll}
\hline Subgroup analysis for BB users $(\boldsymbol{n})$ & CES-D $\geq 8$ MOR $^{\boldsymbol{a}}$; $(\mathbf{9 5 \%}$ confidence intervals); $\boldsymbol{p}$ value \\
\hline $71-90(6117)$ & $1.48(1.06-2.07) ; 0.02$ \\
$>90(678)$ & $4.72(1.52-14.7) ;<0.01$ \\
\hline
\end{tabular}

${ }^{a}$ MOR model-adjusted odds ratio accounting for gender, living status, education, and smoking history (all significant at 0.05 level). Non-significant variables were excluded from multivariable models using a backward elimination method.

$b$ Defined as mean blood pressure (BP) of three measurements with systolic BP > $140 \mathrm{mmHg}$; and/or diastolic $\mathrm{BP}>90 \mathrm{mmHg}$. 


\section{Table 4}

Examining the association of BB and depressive symptoms (CES-D 28 ), according to medication properties, with users of other classes and non-medicated as controls: $(n=14,195)$.

\begin{tabular}{lll}
\hline Exposure (n) $^{\boldsymbol{a}}$ & MOR $^{\boldsymbol{b}}$ and 95\% CI & $\boldsymbol{p}$ value \\
\hline Lipophilic BB (650) & $1.39 ; 1.10-1.75$ & $<0.01$ \\
Hydrophilic BB (846) & $1.26 ; 1.03-1.56$ & 0.02 \\
$\beta$ 1-selective (1276) & $1.31 ; 1.10-1.56$ & $<0.01$ \\
Non-selective (276) & $1.52 ; 1.08-2.13$ & 0.01 \\
Propranolol (144) & $1.56 ; 0.99-2.48$ & 0.05 \\
Metoprolol (434) & $1.40 ; 1.06-1.84$ & 0.01 \\
Atenolol (794) & $1.21 ; 0.98-1.51$ & 0.07 \\
\hline
\end{tabular}

äBB's were classified in subgroups according to properties following Poirier, 2014 [18] as follows:

Lipophilic BB's: (i.e. pindolol, propranolol, timolol, and metoprolol)

Hydrophilic BB's: (i.e. atenolol, carteolol, nadolol, sotalol, and labetalol)

Selective BB: (i.e. atenolol, bisoprolol, labetalol, metoproplol, and nebivolol)

N selective BB: (i.e. propranolol, carvedilol, nadolol, timolol, pindolol, and sotalol).

$b$

Accounting for gender, living status, education, and smoking history (all significant at 0.05 level). Non-significant variables were excluded from multivariable models using a backward elimination method. 